\title{
Dynamically Adaptive Parsons Problems
}

\author{
Barbara J. Ericson \\ Georgia Institute of Technology \\ 801 Atlantic Drive \\ Atlanta, GA, 30332, USA \\ $+1-404.385 .2107$ \\ ericson@cc.gatech.edu
}

\begin{abstract}
Parsons problems are code segments that must be placed in the correct order with the correct indention. Research on Parsons problems suggests that they might be a more effective and efficient learning approach than writing equivalent code, especially for time-strapped secondary teachers. I am exploring this hypothesis with empirical experiments, observations, and log file analyses. Our research team also plans to modify the opensource js-parsons software to allow Parsons problems to be dynamically adaptive, which means that the difficulty of the problem will change based on the user's past performance. I plan to compare dynamically adaptive Parsons problems to the current non-adaptive Parsons problems to determine which learners prefer and to see if solving dynamically adaptive Parsons problems leads to more efficient and effective learning than solving non-adaptive Parsons problems.
\end{abstract}

\section{General Terms}

Design, Experimentation, Measurement

\section{Keywords}

Parsons problems; Learning programming; Online learning; Ebook; Adaptive Learning

\section{RESEARCH SITUATION}

I have just completed the $4^{\text {th }}$ year of my Human-Centered Computing (HCC) $\mathrm{PhD}$ program. I am working on my $\mathrm{PhD}$ parttime while working full-time. I passed my written and oral qualifying exams in the spring of 2014. I passed my proposal in December of 2015.

My research group is creating and testing features for e-books to help people learn programming. One of the features that I have focused on is Parsons problems [8]. Parsons problems are code segments that are mixed up and have to be placed into the correct order and may have to be indented correctly as well. Parsons problems can also have extra code segments, called distractors, which are not needed in a correct solution. The distractor code segments can be displayed in a random order along with the correct code segments or can be displayed paired with the correct code segments.

Permission to make digital or hard copies of part or all of this work for personal or classroom use is granted without fee provided that copies are not made or distributed for profit or commercial advantage and that copies bear this notice and the full citation on the first page. Copyrights for third-party components of this work must be honored. For all other uses, contact the Owner/Author. Copyright is held by the owner/author(s).

ICER '16, September 08-12, 2016, Melbourne, VIC, Australia

ACM 978-1-4503-4449-4/16/09.

http://dx.doi.org/10.1145/2960310.2960342
I created 11 Parsons problems for a chapter of the How to Think Like a Computer Scientist - Interactive Edition Python e-book. Teachers that I observed solving the Parsons problems said that they wanted the Parsons problems to be harder. However, log file analysis of student use of the ebook showed that while the majority of students got most of the Parsons problem right in one to two tries, there were four questions that took three to nine tries for $75 \%$ of the people to solve. Some students had as many as 109 tries before correctly solving one problem. We also found that for some of the easier problems $50 \%$ of the students, who gave up, did so after only 2 tries. For harder problems $50 \%$ of the students, who gave up, did so after six to nine tries.

I also created Parsons problems for an ebook for CS Principles teachers and another one to help students prepare for the Advanced Placement Computer Science A exam. Advanced Placement courses are high school (secondary) courses intended to be equivalent to introductory university courses.

\section{CONTEXT AND MOTIVATION}

National Science Foundation (NSF) wants to prepare 10,000 United States of America high school teachers to teach a new course, Advanced Placement (AP) Computer Science Principles (CS-P), by the beginning of the 2016-2017 school year. This effort is called the CS $10 \mathrm{~K}$ effort. Many researchers in the computer science education community are focusing on this effort, including the members of the CS Learning $4 \mathrm{U}$ project at Georgia Tech.

The new AP CS-P course covers several big ideas in computing, including programming. It would be very expensive and difficult to provide face-to-face professional development for thousands of teachers. Much of the professional development will have to be done using distance learning.

One of the difficulties in preparing teachers to teach programming is that programming can be difficult to learn. Reported failure rates in introductory computer science courses at the university level are as high as $90 \%$ [2]. A large multi-institutional study found that many undergraduate students can't program as well as expected after either their first two computer science courses [5].

The pace of on-line learning can be problematic for teachers. A study of adult learners in two online introductory computer science university courses found that success in the course was dependent on having sufficient time to dedicate to the course work and that working adults often did not have sufficient time [1]. Students had difficulty in learning to program and the time it took to create working programs was unpredictable. Even simple syntax errors like having a comma out of place could take hours to fix [1]. 
Studies of expert programmers show that experts recognize and apply many "programming plans" [7]. Teachers who are new to programming will need to learn these "programming plans". Soloway and Guzdial recommend using scaffolding to support learners as they learn a new task [8]. Parsons problems are a type of scaffolding.

\section{BACKGROUND \& RELATED WORK}

One way to make the learning of computer science more efficient and effective is to reduce the amount of time that beginners struggle with syntactic and semantic errors. One possible approach is to use Parsons problems [6] instead of requiring learners to write lots of code. There are several variants of Parsons problems, such as including extra code as distractors and pairing the distractor and correct code.

Work in this area [3] has found that Parsons problem scores highly correlate with code writing scores. This means that Parsons problems might be a more effective and efficient way to learn than the traditional approach, which requires beginners to spend an unpredictable number of hours writing code. Denny, Luxton-Reilly et al. suggested that students should be able to solve the Parsons problems more quickly than writing the equivalent code, but they did not test this hypothesis. They also tested several variants of Parsons problems and found that students had the most trouble with Parsons problems with one distractor per code block with all blocks in a random order and had the easiest time solving Parsons problems when they were given the structure of the solution (the number of lines in each code block and the indention). Students also had an easier time when the distractors were paired with the correct code.

In the e-books we are using an open-source tool called js-parons. Researchers using this tool have found that even though Parsons problems remove syntax errors, users can get stuck and repeat the same incorrect solution [4]. Some users also exhibit a "trial and error" approach [4]. They drag a single code block into the solution area and ask for feedback to see if it is in the correct place.

\section{STATEMENT OF THESIS/PROBLEM}

Do paired distractor dynamically adaptive Parsons problems help beginners learn programming more effectively and efficiently compared to writing code or fixing code? Our research group has modified js-parsons to allow for paired distractors. Our research group also plans to extend the js-parsons tool to allow for dynamically adaptive Parsons problems, which means that the difficulty of the problem will change based on the user's performance.

\section{DISSERTATION STATUS}

I created one set of Parsons problems and observed teachers solving those problems. The teachers wanted the Parsons problems to be harder. However, log file analysis of students solving the same problems revealed that some students had a great deal of difficulty solving the Parsons problems. By making the Parsons problems dynamically adaptive we should improve learning by keeping the learner in the Zone of Proximal Development.
I created experimental materials for comparing solving Parsons problems that have paired distractors with syntactic and semantic errors, against fixing code that contains the same syntactic and semantic errors, and also against writing the equivalent code. I plan to run that experiment this summer. Our research group is changing js-parsons to allow dynamically adaptive Parsons problems this summer. I plan to observe teachers solving current Parsons problems and dynamically adaptive ones in the fall of 2016. In the spring of 2016 I plan to compare the effectiveness and efficiency of learning from current Parsons problems versus dynamically adaptive ones. I hope to defend by the end of 2017 .

\section{EXPECTED CONTRIBUTIONS}

Research on Parsons problems suggests that they might be a more effective and efficient learning approach than writing equivalent computer programs. However, more research is needed to solidify this claim and to test if dynamically changing the difficulty of the Parsons problems results in more effective and efficient learning. If this research helps teachers learn programming on-line in an efficient and effective manner, it could provide a scalable solution to allow the US to prepare 10,000 computing teachers by $2016-2017$.

\section{ACKNOWLEDGEMENT}

This work is supported by the National Science Foundation grant CNS-1138378.

\section{REFERENCES}

[1] Benda, K. and A. Bruckman (2012). "When life and learning do not fit: Challenges of workload and communication in introductory computer science online." ACM Transactions on Computing Education.

[2] Bennedsen, J. and M. E. Caspersen (2007). "Failure rates in introductory programming." SIGCSE Bull. 39(2): 32-36.

[3] Denny, P., et al. (2008). Evaluating a New Exam Question: Parsons Problems. International Computing Education Research Conference. Sydney, Australia, ACM.

[4] Helminen, J., et al. (2012). How Do Students Solve Parsons Programming Problems? - An Analysis of Ineraction Traces. International Computing Education Research Conference. Aukland, New Zealand, ACM: 119-126.

[5] McCracken, M., et al. (2001). "A multi-national, multiinstitutional study of assessment of programming skills of first-year CS students." SIGCSE Bull. 33(4): 125-180.

[6] Parsons, D. and P. Haden (2006). Parson's programming puzzles: a fun and effective learning tool for first programming courses. Proceedings of the 8th Australasian Conference on Computing Education - Volume 52. Hobart, Australia, Australian Computer Society, Inc.: 157-163.

[7] Soloway, E. and K. Ehrlich (1984). "Empirical Studies of Programming Knowledge." IEEE Transactions on Software Engineering SE-10(5): 595-609.

[8] Soloway, E., et al. (1994). "Learner-Centered Design: The Challenge For HCI In The 21st Century.” Interactions 1(2) 36-48 